\title{
An investigation of the relationship between the transdermal drug delivery and membranes fludity by multiphoton imaging of Laurdan
} Yen Sun ${ }^{1}$, Jiun-wen Su${ }^{1}$, Sun-Jan Lin ${ }^{2}$, Shiou-Hwa Jee ${ }^{2,3}$, Chen-Yuan Dong ${ }^{1 *}$.

${ }^{1}$ Department of Physics, National Taiwan University, Taipei, Taiwan Republic of China, ${ }^{2}$ National Taiwan University Hospital, Taipei, Taiwan Republic of China, ${ }^{3}$ College of Medicine, National Taiwan University, Taipei, Taiwan Republic of China Tel: 3366-5155 Fax:3366-5244 E-mail: cydong@phys.ntu.edu.tw *Corresponding author: Chen-Yuan Dong

Abstract Multiphoton microscopy is a three-dimensional imaging technique with a number of advantages. For example, multiphoton microscopy can image tissues up to a depth of a few hundred micrometers, at high resolution and with improved contrast. In this work, we used multiphoton microscopy in investigating the actions of the chemical enhancer oleic acid in transdermal drug delivery. Technique of generalized polarization (GP) imaging and polarization (P) imaging associate with Laurdan to monitor the lipid layer changes caused by the addition of oleic acid. Our study will help to develop more effective methodologies in the delivering of molecules across biological barriers. Methods and Results Transdermal drug delivery utilizes passive diffusion of drugs across the skin as the delivery mechanism. It many advantages including a steady blood-level profile of drug concentration, being painless, use-friendly, and convenient. However, to efficiently delivery doses of therapeutic dugs through the skin into systemic circulation is very difficult due to the penetration barrier properties of the stratum corneum. Many investigations have been performed to improve skin permeation of drugs into stratum corneum by using several chemical or physical techniques such as the application of chemical enhancers, iontophoresis, and electroporation. However, the mechanism of these techniques is not completely understood. In this work, we demonstrate that by combining polarization and generalized polarization imaging techniques, we can gain a better understanding of the mechanism of the oleic acid in transdermal drug delivery.

We combined two multiphoton imaging techniques for characterizing changes in the physical and chemical properties of intercellular lipid layers of the skin: polarization (P) and generalized polarization (GP) imaging. Polarization (P) and generalized polarization (GP) are respectively defined as:

$$
P=\frac{I_{I I}-I_{\perp}}{I_{I I}+I_{\perp}} \quad \text { and } \quad G P=\frac{I_{440}-I_{490}}{I_{440}+I_{490}}
$$

The polarized fluorescence emission is characterized by measuring the fluorescence parallel $\left(I_{\mathrm{II}}\right)$ and perpendicular $\left(I_{1}\right)$ to the excitation polarization. Another technique of GP imaging can be used to characterize the microenvironment of the fluorescent probe Laurdan. In this approach the GP value is an indication of the spectral shift of Laurdan $\left(\mathrm{I}_{440}\right.$ in Eq 1 represents the emission intensity at $440 \mathrm{~nm}$ while $\mathrm{I}_{490}$ represents the emission intensity at $490 \mathrm{~nm}$ ). Since Laurdan emission becomes redshifted in a polar environment, a decrease in the GP value indicates a more polar solvent molecules penetrating into the skin structures. 


\section{THP-26(T5)}

To evaluate the effectiveness of polarization and GP imaging in detecting structural changes in the lipid layer we imaged the same area of the skin for polarization and GP imaging (with and without oleic acid OA). The results are shown in Fig. 1.

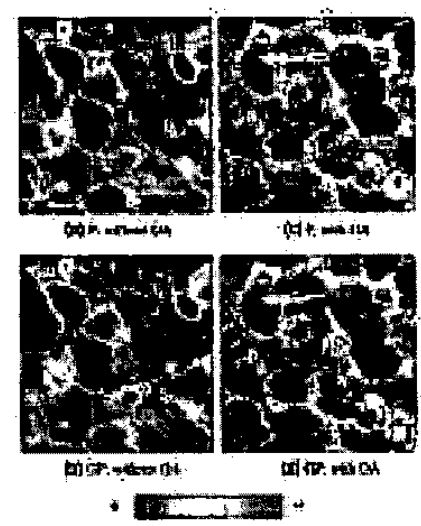

Fig. 1. Laurdan skin polarization images in the (a) absence and (c) presence of OA. Laurdan GP images (b) without and (d) with $\mathrm{OA}$ (Imax=1, Imin=-1). In (b) and (d), two areas are chosen for GP analysis. In (b), the GPs for areas b1 and b2 are $0.37 \pm 0.15$, $0.27 \pm 0.14$, respectively. In (d) the GPs for $\mathrm{d} l$ and $\mathrm{d} 2$ are, respectively, $-0.08 \pm 0.13$ and $0.11 \pm 0.11$.

Fig. 1 shows that the addition of oleic acid leads to a reduction of GP. Therefore our GP results are consistent with the fact that the addition of $\mathrm{OA}$ disturbs the lipid packing in the skin and allows water molecules to penetrate the lipid layers of the skin. Analysis of the polarization data (to be presented at the meeting) also shows the addition of oleic acid leads to a randomization of lipid packing in the skin.

Conclusion In conclusion, we demonstrated that polarization and generalized polarization imaging may be combined to monitor structural changes in the lipid structures of skin. Polarization imaging reveals the molecular orientation in biological structures, and GP imaging provides information on changes in the molecular microenvironment. A combination of multiphoton polarization and GP imaging is a useful technique for understanding physical and chemical changes in biological systems.

\section{References}

1. W. Denk, J. H. Strickler, and W. W.Webb, Science 248, 73 (1990).

2. P. T. C. So, C. Y. Dong, B. R. Masters, and K. M. Berland, Ann. Rev. Biomed. Eng. 2, 399 (2000).

3. B. Yu, C. Y. Dong, P. T. C. So, D. Blankschtein, and R. Langer, J. Invest. Dermatol. 117, 16 (2001).

4. B. Yu, K. H. Kim, P. T. C. So, D. Blankschtein, and R. Langer, J. Invest. Dermatol. 118, 1085

5. R. O. Potts and M. L. Francoeur, Proc. Nat. Acad. Sci. USA 87, 3871 (1990).

6. L. A. Bagatolli and E. Gratton, Biophys. J. 78, 290 (2000).

7. Y. Sun, J. W. Su, W. Lo, S. J. Lin, S. H. Jee, and C. Y. Dong, Opt. Express 11, 3377 (2003).

8. J. A. Boustra, F. E. R. Dubbelaar, G. S. Gooris, and M.vPonec, Acta Derm. Venereol. Suppl. 208, 23 (2000).

9. K. C. Madison, J. Invest. Dermatol. 121, 231 (2003).

10. J. R. Lakowicz, Principles of Fluorescence Spectroscopyv(Plenum, New York, 1999. 